\title{
AN EXPERIMENTAL COMPARISON OF NONSWIRLING AND SWIRLING FLOW IN A CIRCULAR-TO-RECTANGULAR TRANSITION DUCT
}

\author{
B.A. Reichert* and W.R. Hingst ${ }^{\dagger}$ \\ National Aeronautics and Space Administration \\ Lewis Research Center \\ Cleveland, Ohio 44135 \\ and \\ T.H. Okiishi ${ }^{\ddagger}$ \\ Iowa State University \\ Ames, Iowa 50010
}

\begin{abstract}
Circular-to-rectangular transition ducts are used as exhaust system components of aircraft with rectangular exhaust nozzles. Often, the incoming flow of these transition ducts includes a swirling velocity component remaining from the gas turbine engine. Previous transition duct studies have either not included inlet swirl or when inlet swirl was considered, only overall performance parameters were evaluated. This paper explores circular-torectangular transition duct flows with and without inlet swirl in order to understand the effect of inlet swirl on the transition duct flow field and to provide detailed duct flow data for comparison with numerical code predictions.

A method was devised to create a swirling, solid body rotational flow with minimal associated disturbances. Coefficients based on velocities and total and static pressures measured in cross stream planes at four axial locations within the transition duct, along with surface static pressure measurements and surface oil film visualization, are presented for both nonswirling and swirling incoming flow. In both cases the inlet centerline Mach number was 0.35 . The Reynolds number based on the inlet centerline velocity and duct inlet diameter was $1,547,000$ for nonswirling flow and $1,366,000$ for swirling flow. The maximum swirl angle was $15.6^{\circ}$. Two pair of counter-rotating side wall vortices appeared in the duct flow without inlet swirl. These vortices were absent in the swirling incoming flow case.
\end{abstract}

\section{Introduction}

Nonaxisymmetric exhaust nozzles are employed on high performance aircraft to improve performance. Rectangular exhaust nozzles require a circular-to-rectangular transition duct to connect the engine and nozzle. To maximize nozzle performance the transition duct should minimize transverse velocity components at the duct exit and the total pressure loss in the duct.

In typical exhaust component applications the incoming flow to the circular-to-rectangular transition duct

\footnotetext{
'Research Engineer, Inlet, Duct, and Nozzle Flow Physics Branch.

${ }^{\dagger}$ Research Engineer, Inlet, Duct, and Nozzle Flow Physics Branch, Member AIAA.

${ }^{\ddagger}$ Professor and Chair, Mechanical Engineering Department, Member AIAA
}

is turbulent, subsonic, and not fully developed. Often, the incoming flow includes a significant rotational velocity component. The tcrm swirl refers to this rotational velocity component, which remains from the engine turbine. Representative studies of turbine exit flow angles ${ }^{1-3}$ have shown that swirl often exists at turbine design operating conditions, and may be as great as $30^{\circ}$ or morc at off-design operating conditions. Inlet swirl can significantly alter the flow field throughout the transition duct.

Previous researchers have experimentally explored the acrodynamics of circular-to-rectangular transition ducts. Patrick and McCormick ${ }^{4,5}$ recorded values of total pressure, mean velocity, and three normal Rcynolds stress components at the inlet and exit planes of two different circular-to-rectangular transition ducts. Miau et al.$^{6,7}$ measured mean velocities and turbulence intensities at the inlet and exit of threc circular-lo-rectangular transition ducts. In a benchmark study, Davis and Gessner ${ }^{8}$ measured static pressures, mean velocilies, and all six Reynolds stress components in three cross stream planes within a circular-to-rectangular transition duct. Each of these studies involved incompressible flow without inlet swirl.

Burley et al. ${ }^{9.10}$ testcd five circular-to-rectangular transition ducts including one with swirl vanes installed upstream of the duct inlet. These measurements were, however, limited to values of surface static pressure, thrust ratio performance parameter, and discharge coefficient.

The objective of the rescarch described in this paper was two-fold; to ascertain the effect of inlet swirl on the transition duct flow ficld and to provide a set of detailed data for validating numerical code predictions of transition duct flows with and without inlet swirl. A new method for swirl generation was employed to add a swirling velocity component to the flow just upstream of the transition duct. The intent of the swirl generator was to approximate a solid body rotational flow free of wakes and other disturbances. Presented are cocfficients based on detailed measurements of velocity, total pressure and static pressure, acquired in four cross stream planes within a circular-to-rectangular transition duct, with and without inlet swirl. In addition, surface static pressure and surface oil film visualization results are presented. 


\section{Experimental Facilitics}

\section{Circular-to-Rectangular Transition Duct}

Fig. 1 shows a drawing of the lower half of the circular-to-rectangular duct that was tested and the coordinate system used. This transition duct is a member of a family of ducts designed at the NASA Lewis Research Center. In the $y z$-plane through cach cross section, the surface of the ducts satisfies Eq. (1).

$$
\left(\frac{y}{r_{y}}\right)^{n}+\left(\frac{z}{r_{z}}\right)^{n}=1
$$

The parameters $r_{y}, r_{z}$ and $n$, which specify the exact geometry, are all functions of the axial distance, $x$. The cross-sectional shape of these transition ducts becomes more rectangular as the value of the exponent $n$ increases but they are never truly rectangular. This was done to provide the smooth boundary required by some CFD methods. The circular-to-rectangular ducts studied in Refs. 4, 5, 8-10 are all members of this family of transition ducts. The values of the parameters for the transition duct constructed for this study are contained in Ref. 11.



Fig. 1 Circular-to-rectangular transition duct.

The transition region of the duct is where the cross scction in the $y z$-plane varies with axial distance. The ratio of the length of the transition region $L$ to the inlet diameter $D$ is $L / D=1.5$. The transition region lies within $1.0 \leq x / D \leq 2.5$. The ratio of major to minor axis lengths at the duct exit, referred to as the aspect ratio, is $r_{y} / r_{z}=3.0$. The cross-sectional areas at the inlet and exit are cqual. In the transition region the cross-sectional area increases to as much as 1.15 times the inlet area. The inlet diameter of the duct is $20.42 \mathrm{~cm}$.

The transition duct was constructed in two halves joined at the $x y$-plane. Each half was machined from a single piece of aluminum with a numerically controlled mill. This transition duct was also used to produce mold to fabricate an identical fiberglass duct used by Davis and $\mathrm{Gessner}^{8}$ for measurements made at the University of Washington.

\section{Swirl Generator}

The function of the swirl generator is to produce a solid body rotational flow superimposed on a uniform flow. Ideally, this rotating flow is free of wakes or other disturbances caused by the mechanism which creates the rotation. In cylindrical coordinates this velocity field is described by Eq. (2), where $V_{\text {centerline is the velocity }}$ at the swirl generator centerline and $\Omega$ is the angular velocity of the solid body rotation.

$$
V_{x}=V_{\text {centerline }}, V_{\theta}=\Omega r, V_{r}=0
$$

An example of this flow is represented in Fig. 2. The swirl angle, $\phi$, is given by Eq. (3).

$$
\phi=\arctan \left(\frac{V_{\theta}}{V_{x}}\right)
$$

This is the angle of the flow relative to the $x$-axis measured by a stationary observer.

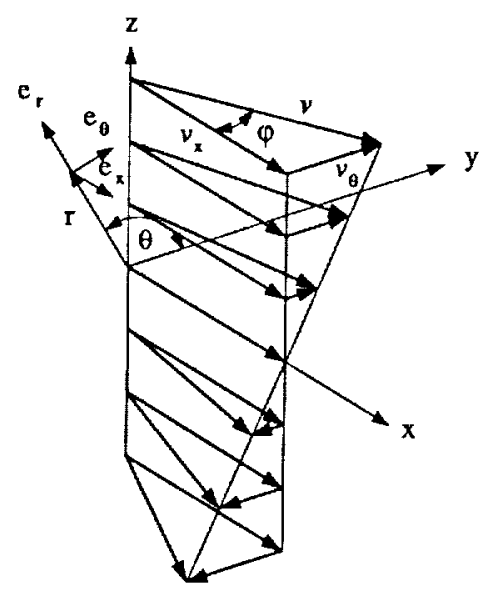

Fig. 2 Solid body rotational flow superimposed on uniform flow.

Fig. 3 shows a schematic of the swirl generator that was conceived, designed and constructed for this study. This generator employs both stationary blades and a rotating pipe. The flow coming into the stationary blades is nominally uniform and one-dimensional. The blades create a near solid body rotational flow at an angular velocity that depends on both the axial flow velocity and the blade camber angle, which could be varied independently.

Immediately downstream of the stationary blades is the rotating pipe. The honeycomb and screen are fixed to the rotating pipe and these components rotate as one assembly. The honcycomb serves as a rotor that drives the rotating pipe. Additionally, the honeycomb reinforces the near solid body rotation initiated by the 


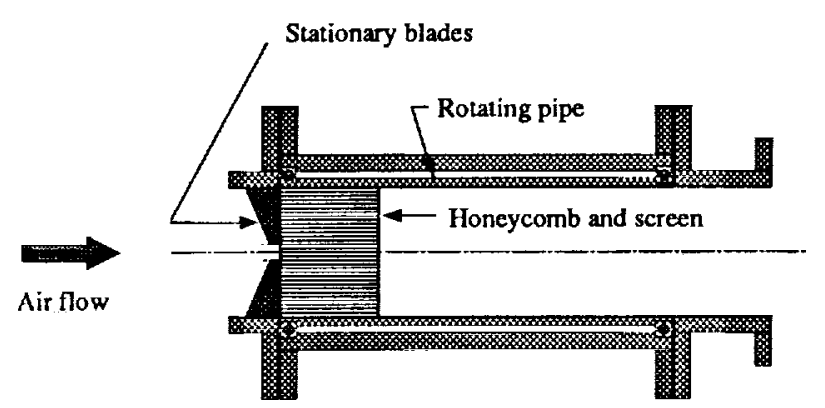

Fig. 3 Swirl generator schematic.

stationary blades and dissipates the wakes created by the stationary blades. A screen is located downstream of the honeycomb to dissipate the wakes formed by the honeycomb. The final section of the swirl generator is the stationary exit section. This section functions as a stationary component to which the transition duct may be attached.

The maximum ideal swirl angle $\phi_{\text {max }}$ represents the swirl angle at the rotating pipe wall $(r=R)$ excluding the boundary layer deceleration of the axial velocity component, and is given by Eq. (4). For the results presented in this paper $\phi_{\max }=15.6^{\circ}$. Additional details about the swirl generator design, construction, operation and performance are contained in Ref. 11.

$$
\phi \max =\arctan \left(\frac{\Omega R}{V_{\text {centerline }}}\right)
$$

Test Facility

The tests were conducted at the NASA Lewis Research Center using the Internal Fluid Mechanics Facility. This facility was designed to support research on a variety of intemal flow configurations. Air could be supplied from either the test cell or from a continuous source of pressurized air. After passing through a large scttling chamber containing honeycomb and screens the flow proceeds through an axisymmetric contraction having an area ratio of 57:1. The flow passes from the contraction through either a straight pipe to provide a nonswirling uniform incoming flow to the transition duct, or the swirl generator to provide a swirling incoming flow. After passing through the transition duct the flow is exhausted into a discharge plenum which is continuously evacuated by central exhauster facilities.

\section{Experimental Methods and Results}

All the results presented in this section are nondimensional. Total and static pressures are presented as pressure coefficients, having been nondimensionalized as indicated in Eqs. (5) and (6). Velocity vectors were divided by the local sonic velocity $c$ to yield a Mach vector and then normalized by the centerline Mach number, as shown in Eq. (7). Bold type in Eq. (7) indicates vector quantities. In Eqs. (5) through (7) the reference variables, subscripted centerline $(r=0)$ or wall $(r=R)$, were evaluated at a location one radius upstream of the transition duct inlet $(x / D=-0.5)$. This location represents conditions at the exit of the straight pipe or swirl generator or the inlet of the transition duct. A discussion and an analysis of the results are given in the following section titled Discussion and Conclusions.

$$
\begin{gathered}
p_{0}^{*}=\frac{p_{0}-p_{\text {wall }}}{p_{0, \text { centerline }}-p_{\text {wall }}} \\
p^{*}=\frac{p-p_{\text {wall }}}{p_{0, \text { centerline }}-p_{\text {wall }}} \\
\mathbf{M}^{*}=\frac{\mathrm{V} / c}{M_{\text {centerline }}}
\end{gathered}
$$

\section{Test Conditions}

Test conditions were verified by a survey of the flow field one radius upstream of the transition duct inlet. The test conditions for the flow without and with inlet swirl are summarized in Table 1. The Reynolds number is based on the centerline velocity and the transition duct inlet diameter. The difference in Reynolds number between the two cases resulted from a total pressure loss associated with the swirl generator. The boundary layer thickness $\delta_{0.95}$, displacement thickness $\delta^{*}$, momentum thickness $\theta$, and shape factor $H$, were calculated from the survey data. The total temperature for all tests was nominally $274 \mathrm{~K}$.

Table 1 Test conditions for flow without and with inlet swirl

\begin{tabular}{ccc}
\hline & $\begin{array}{c}\text { Without } \\
\text { inlet swirl }\end{array}$ & $\begin{array}{c}\text { With inlet } \\
\text { swirl }\end{array}$ \\
\hline$M_{\text {centerline }}$ & 0.35 & 0.35 \\
$\operatorname{Re}_{D}$ & $1,547,000$ & $1,366,000$ \\
$\phi \max$ & $0.0^{\circ}$ & $15.6^{\circ}$ \\
$\delta_{0.95} / R(\times 100)$ & 8.014 & 20.484 \\
$\delta^{*} / R(\times 100)$ & 1.487 & 4.048 \\
$\theta / R(\times 100)$ & 1.034 & 2.370 \\
$H$ & 1.438 & 1.707 \\
\hline \hline
\end{tabular}

Surface Oil Film Visualization

A surface oil film visualization technique was used to obtain preliminary information about the flow field near the surface of the transition duct. The results of this initial "quick look" were used to guide subsequent aerodynamic 


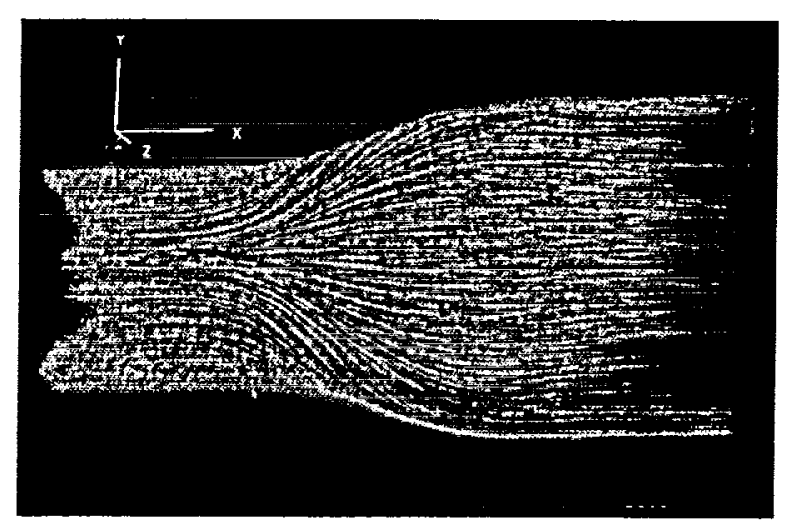

a)

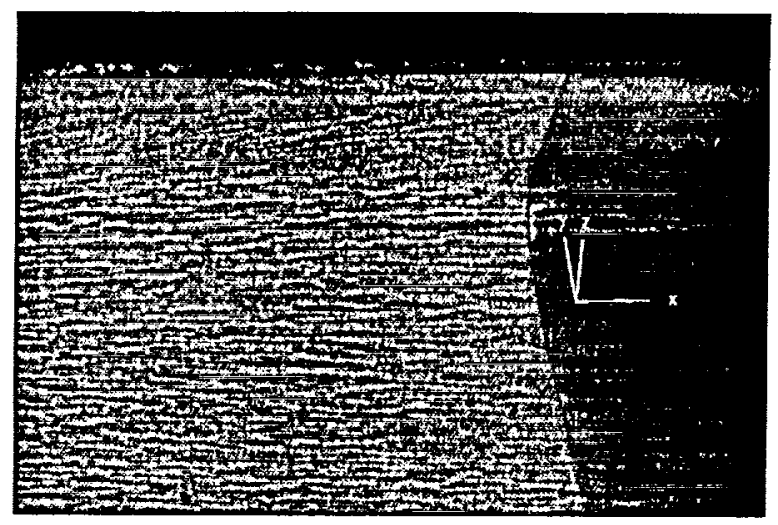

b)

Fig. 4 Surface oil film visualization for nonswirling incoming flow.

measurements. An analysis by Squire $^{12}$ showed that the most significant parameter affecting the validity of surface oil film visualization results is the ratio of the viscosity of the boundary layer fluid to the viscosity of oil. For small viscosity ratios and thin oil films the boundary conditions are not materially changed by the presence of the oil film. Furthermore, for small viscosity ratios and large local shear stresses the direction indicated by the oil flow will accurately represent the flow ficld direction near the surface. Where the shear stress is small (e.g. near separation) the oil film motion becomes influenced by near surface pressure gradients and may not accurately reflect the near surface flow field direction.

The surface oil film visualization technique used was similar to the method described by Jurkovich, Greber and Hingst $^{13}$. The resulting air to oil viscosity ratio for the oil used was approximately $10^{-6}$. A commercial dye which fluoresces when illuminated with ultraviolet light was mixed with the oil. Using fluorescent dye with ultraviolet illumination produces brighter images and reduces glare from the duct surface.

At the beginning of a visualization test, the upper half of the transition duct was removed and the oil and dye mixture was applied to the lower duct surface. Care was taken in applying the oil and dye mixture to avoid a visible pattern. The upper half of the duct was replaced

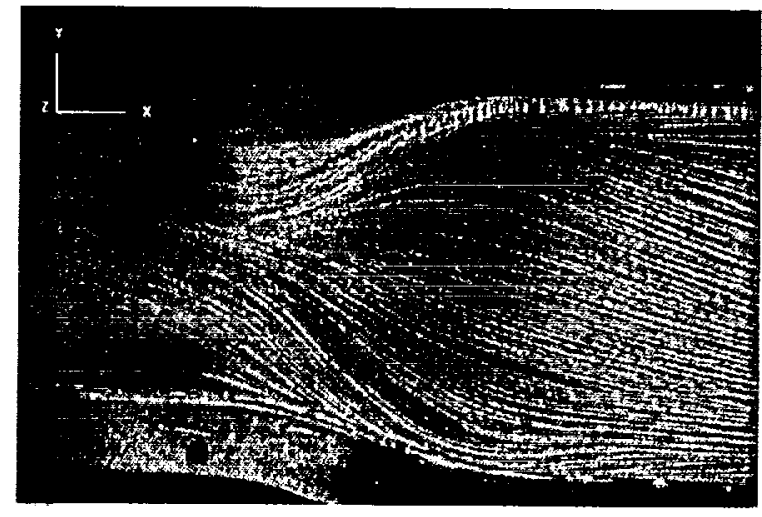

a)

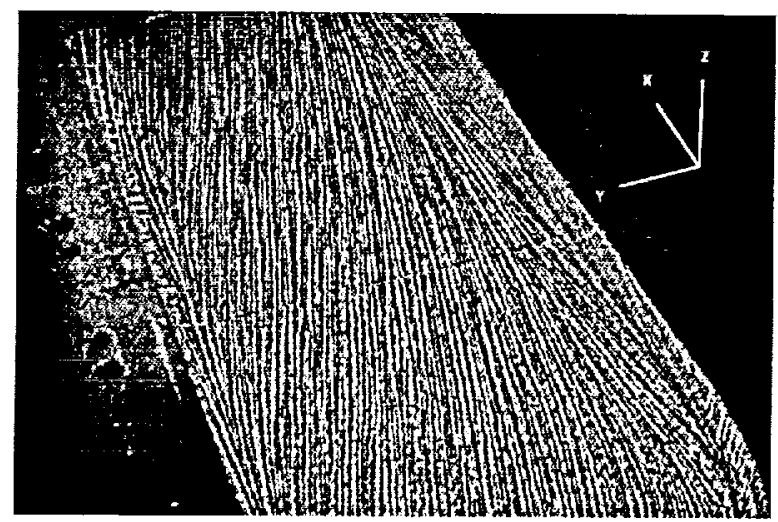

b)

Fig. 5 Surface oil film visualization for swirling incoming flow.

and the test facility was started, operated at test conditions for several minutes, then shut down. The upper half of the transition duct was subsequently removed and the lower half was illuminated with ultraviolet light to excite the fluorescent dye. The resulting oil flow pattern was photographed with a $35 \mathrm{~mm}$ camera with sufficient exposure time to use the ultraviolet light for illumination.

Fig. 4 shows surface oil film visualization results for flow without inlet swirl from two different views. In both figures the flow is from the left to the right. A local coordinate system is shown in each figure to help establish the orientation of the photographs. These photographs were digitized with an image scanner and then numerically enhanced to improve their contrast. The results for flow with inlet swirl are shown in Fig. 5.

\section{Surface Static Pressure Measurements}

Surface static pressure measurements were made through small $(0.51 \mathrm{~mm})$ tap holes whose axes are oriented normal to the duct surface. There were 50 static pressure taps equally spaced on the duct surface in the $x z$-plane along the broken line shown in Fig. 1 .

Open symbols in Fig. 6 represent the static pressure coefficient $p^{*}$ along the lower surface of the duct for flow with and without inlet swirl. Shown as vertical 


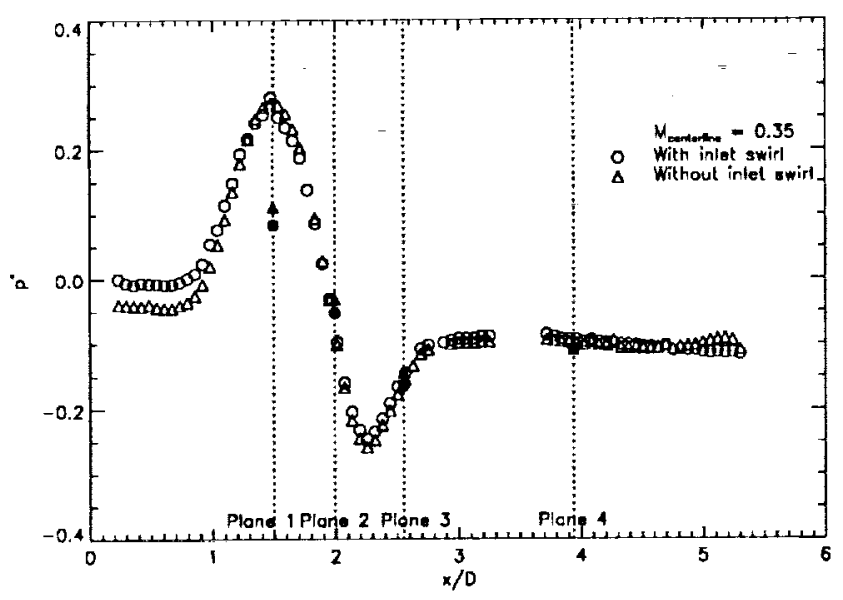

Fig. 6 Surface static pressure coefficient for flow with and without inlet swirl.

broken lines are the locations of the four cross stream measurement planes. The solid symbols on these lines represent the static pressure coefficient measured at the centerline during the acrodynamic surveys.

\section{Five-Hole Probe Measurements}

A calibrated five-hole probe was used to measure air flow velocity, direction, total pressure and static pressure in four cross stream measurement planes in the transition duct shown in Fig. 1. From each pair of total and static pressures a corresponding Mach number was calculated. All five-hole probe measurements were made using the yaw nulling techniquc. Five-hole probe access was provided by openings in the transition duct wall located at the four measurements planes. The axial location of the four cross stream measurement planes is given in Tablc 2 . Approximately 480 five-hole probe measurements were recorded at each measurement plane.

Table 2 Axial location of cross stream measurement planes

\begin{tabular}{ccccc}
\hline \hline Plane & 1 & 2 & 3 & 4 \\
$x / D$ & 1.49 & 1.99 & 2.55 & 3.93 \\
\hline \hline
\end{tabular}

The transition duct is symmetric with respect to the horizontal $x y$-plane and the vertical $x z$-plane. For flow without inlet swirl it would have bcen sufficient to make measurements in only one quadrant at each measurement plane. Measurements were made in two quadrants, howcver, on both sides of the $x z$-plane. The additional quadrant of measurements were made to confirm that the flow field does reflect the symmetry of the transition duct for flow without inlet swirl, and to provide a comparison for the swirling flow case, where measurements in two adjacent quadrants were required.
The results of detailed surveys of the flow in the four cross stream planes within the transition duct are shown in Figs. 7 through 10. The view in Figs. 7 through 10 is looking downstream with the flow from left to right. The cross section of the transition duct is drawn to the same scale in each figure. Measurements were made in the lower half of the transition duct only. The data shown in the upper half of the duct in Figs. 7 through 10 is rotated from the lower half.

Contours of the axial component of $\mathrm{M}^{*}$ at each measurement plane for flow without and with inlet swirl are plotted in Figs. 7a and 9a. Figs. $7 \mathrm{~b}$ and $9 \mathrm{~b}$ show the transverse components of $\mathbf{M}^{*}$. Beneath each figure is a vector labeled $M_{\text {centerline. This is the reference length }}$ scale used for the plots of transverse $\mathrm{M}^{*}$ components. The same reference length has becn used at each measurement plane for both figures. This allows direct comparisons to be made between results at different planes or at different inlet conditions.

Contour plots of the static pressure cocfficient $p^{*}$ at each measurement plane are shown in Figs. 8a and 10a. The same contour levels are used in all plots. The broken lines that appear in the static pressure coefficient contours indicate negative coefficient values. Contour plots of the total pressure coefficient $p_{11}^{*}$ are shown in Figs. $8 \mathrm{~b}$ and $10 \mathrm{~b}$.

\section{Discussion and Conclusions}

Flow Without Inlet Swirl

In this section the nondimensional quantities $p_{0}^{*}, p^{*}, \mathbf{M}^{*}$ are referred to simply as total pressure, static pressure and velocity. The distribution of static pressures was generally attributed to the response of the flow field outside the boundary layer to the changing duct geometry. The change in cross section of the duct forced the flow to converge in the $x z$-plane and diverge in the $x y$-plane. The duct wall deflected the incoming flow, initially parallel to the $x$-axis, away from the $x$-axis in the $x y$-plane and towards the $x$-axis in the $x z$-plane. For the duct flow without inlet swirl, this initially produced a saddle-shaped static pressure distribution in the $y z$-plane, with minimum static pressures near either end of the $y$-axis and maximum static pressures near either end of the $z$-axis. This static pressure distribution can be seen at plane 1 of Fig. 8. The maximum pressures are also observable as the maximum value that appear in the surface static pressure plot shown in Fig. 6 .

Another saddle-shaped static pressure distribution in the $y z$-plane was developed further downstream when the flow was forced by the duct wall back to a direction that is nominally parallel to the $x$-axis in the constant cross section region following the transition region. This static pressure distribution involved maximum static pressures near either end of the $y$-axis and minimum static 


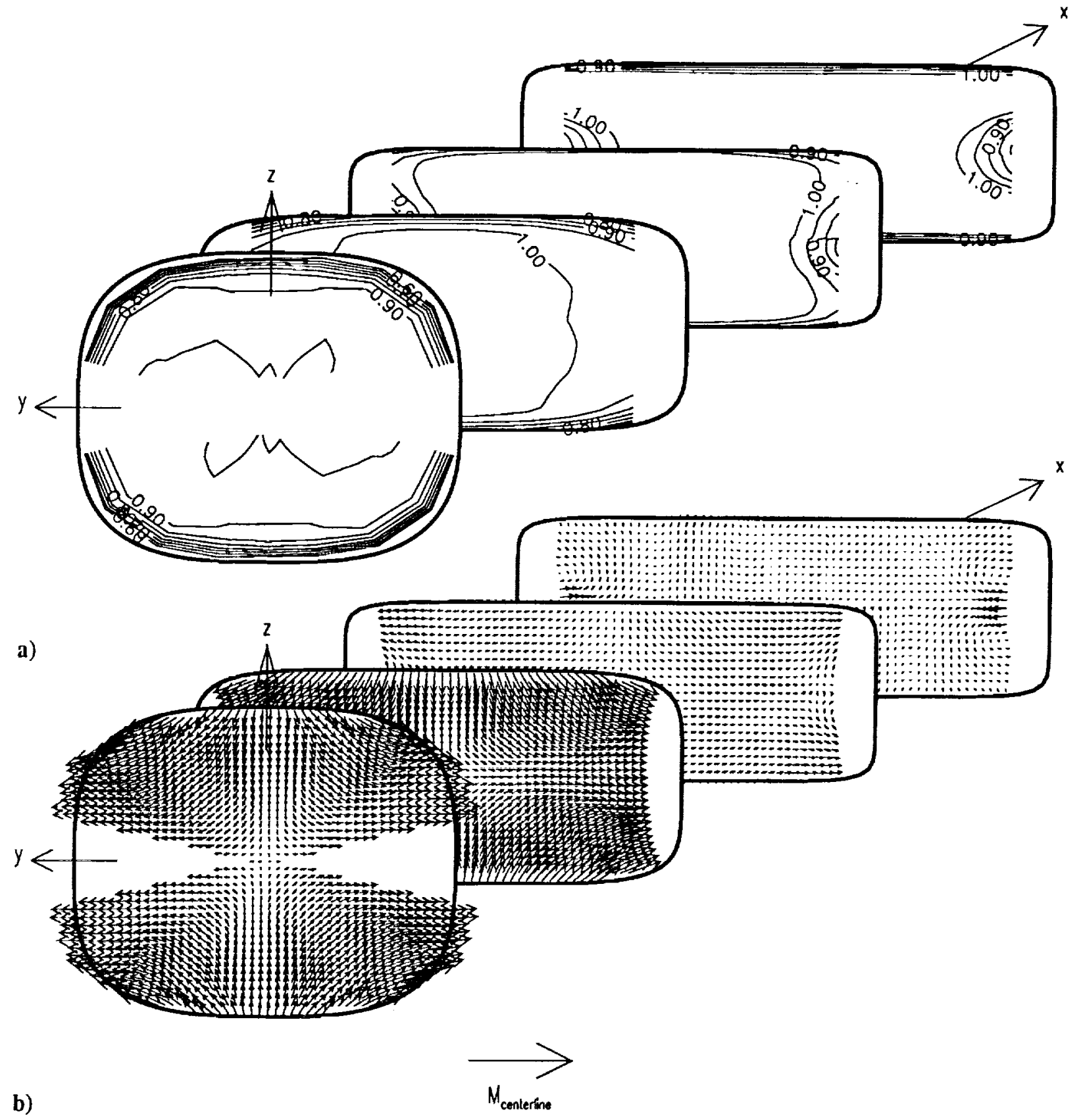

Fig. 7 Axial (a) and transverse (b) components of $\mathrm{M}^{*}$ for flow without inlet swirl.

pressures near either end of the $z$-axis. Evidence of this distribution can be seen in the static pressure distribution at plane 3 of Fig. 8 and as the minimum value of the surface static pressure plot shown in Fig. 6. Miau et al. ${ }^{6,7}$ and Davis and Gessner ${ }^{8}$ also observed this reversal of the static pressure distribution in their surface static pressure measurements.
The location of the maximum and minimum static pressures were reversed between planes 1 and 3 for reasons described above. In order to affect the reversal of static pressure, there must be an intermediate location where the static pressure distribution in the $y z$-plane was nominally flat. The results show the intermediate location must be between planes 1 , at $x / D=1.49$, and 2 , 


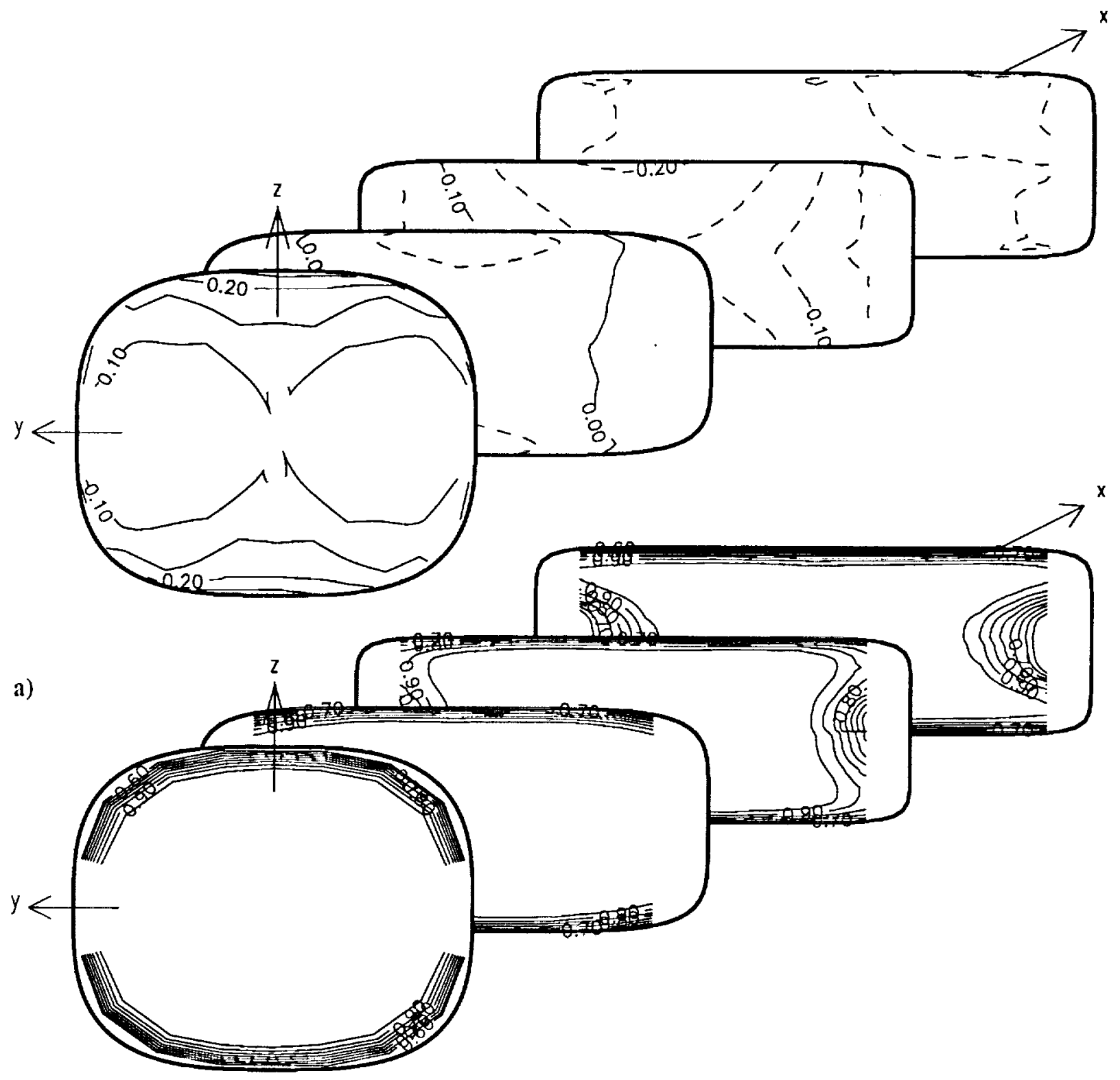

b)

Fig. 8 Static pressure coefficient $p^{*}$ (a) and total pressure coefficient $p_{0}^{*}$ (b) distributions for flow without inlet swirl.

at $x / D=1.99$. The static pressure distribution at plane 2 of Fig. 8 already shows the reversal of the location of the maximum and minimum static pressures, although the gradients are less than those observed at plane 3. The surface static pressure measurements made along axial cross sections in an identical duct by Davis and Gessner ${ }^{8}$ show that the reversal occurs between $x / D=1.60$ and $x / D=1.90$. The overall level of static pressure was higher at planes 1 and 2 because of the increase in crosssectional area at these measurement planes.

The static pressure distribution at plane 4 of Fig. 8 is nominally flat. The slight gradients observed in the static 


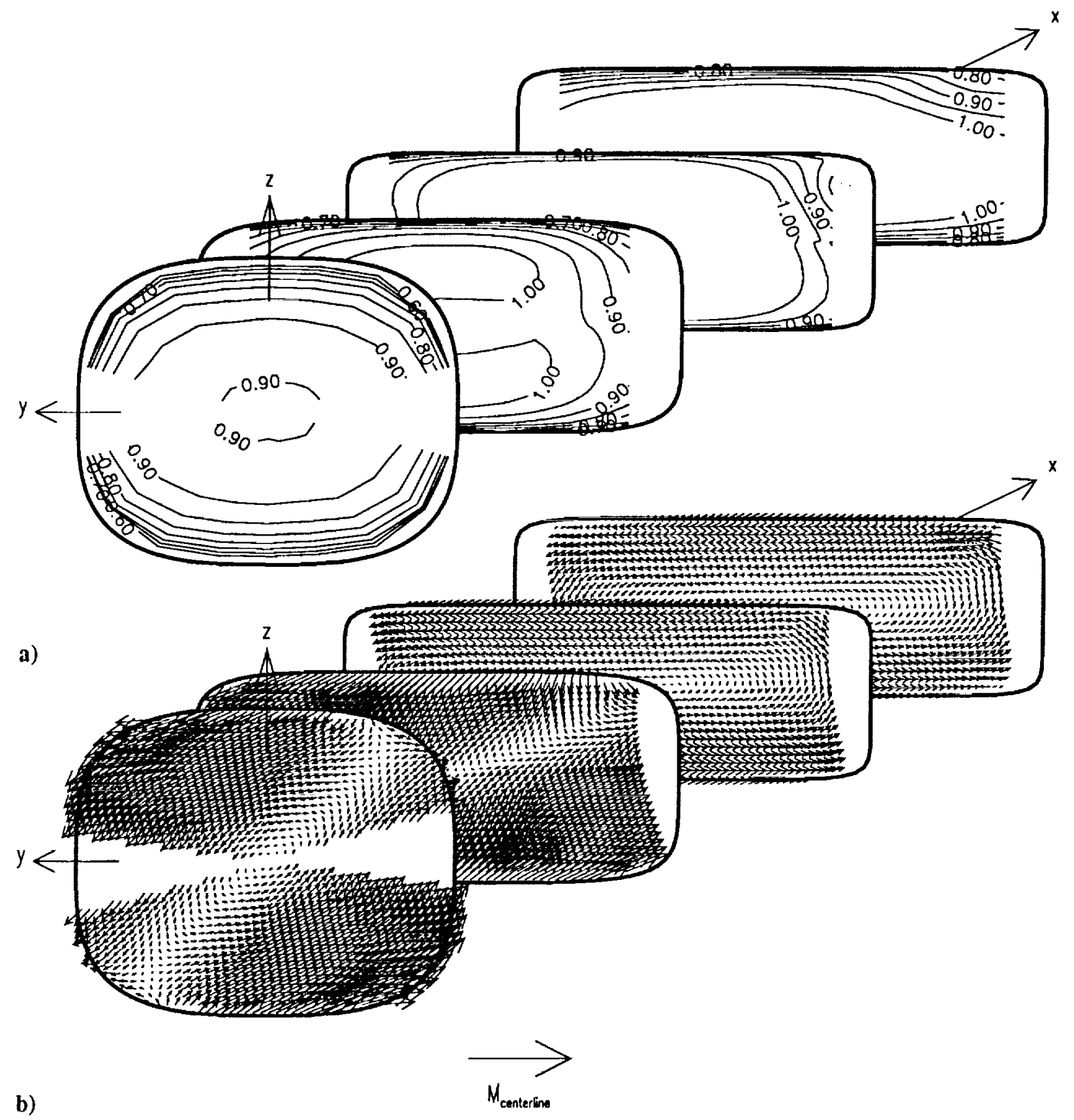

Fig. 9 Axial (a) and transverse (b) components of $\mathrm{M}^{*}$ for flow with inlet swirl.

pressure distribution probably resulted from the upstream influence of the discharge plenum at the transition duct exit, which was an abrupt enlargement.

Because the static pressure distribution was established by the flow field outside the boundary layer where the momentum is greatest, the resulting static pressure distribution can produce significant turning of the flow within the boundary layer where the momentum is less. This is the source of skew-induced secondary flow referred to by Bradshaw. ${ }^{14}$ This can be seen at plane 2 of Fig. 7, where the greatest transverse velocity components appear near the duct surface towards the side walls.

The explanation of skew-induced secondary flows in the proceeding paragraph has its foundation in the vor- 


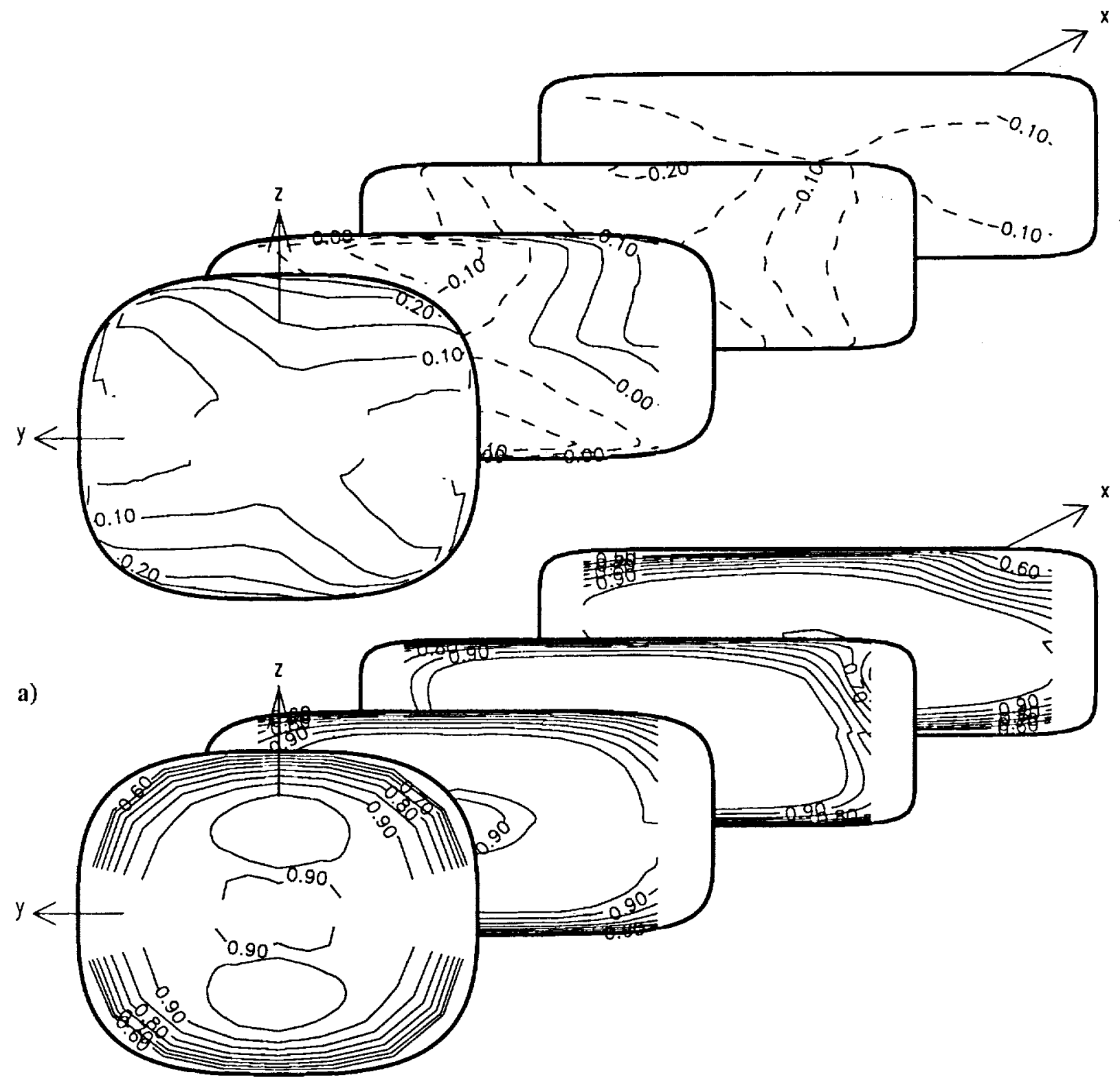

b)

Fig. 10 Static pressure coefficient $p^{*}$ (a) and total pressure coefficient $p_{0}^{*}$ (b) distributions for flow with inlet swirl.

ticity transport equation. The $x$ component of the vorticity transport equation, neglecting viscous and Reynolds stress terms, is given by Eq. (8). The first term in the right hand side of Eq. (8) is referred to as the vortex stretching term, the next two terms are referred to as the vortex tilting terms.

$$
\frac{D \omega_{x}}{D t}=\omega_{x} \frac{\partial u}{\partial x}+\omega_{y} \frac{\partial u}{\partial y}+\omega_{z} \frac{\partial u}{\partial z}
$$

For the idealized case of a simple boundary layer in the $x z$-plane, $u(y), v=0, w=0$, with a cross stream static pressure gradient, $\frac{\partial p}{\partial x}=0, \frac{\partial p}{\partial y}=0, \frac{\partial p}{\partial z} \neq 0$, Eq. (8) reduces to Eq. (9). The right hand side of Eq. (9) 


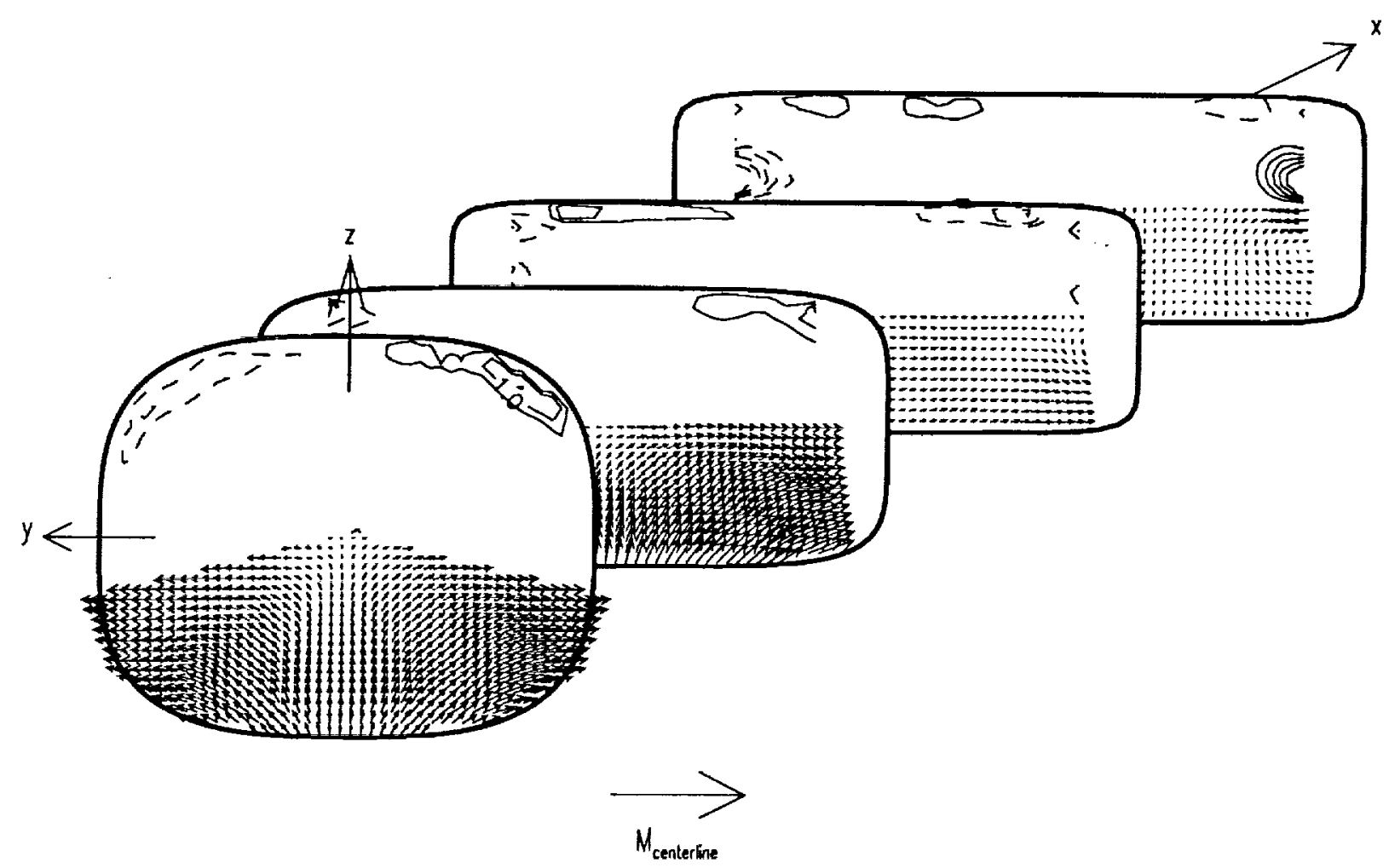

Fig. 11 Axial vorticity and transverse velocity for flow without inlet swirl.

arises from the second term of the right hand side of Eq. (8).

$$
u \frac{\partial \omega_{x}}{\partial x}=\frac{1}{\rho u} \frac{\partial p}{\partial z} \frac{\partial u}{\partial y}
$$

The outward directed flow in the boundary layer near the side walls seen in the transverse velocity plots at planes 1 and 2 was the genesis of the two pair of counterrotating side wall vortices that were clearly present at planes 3 and 4 . Although the static pressure distribution reversed orientation between planes 1 and 2, the second static pressure distribution failed to completely reverse the secondary fluid motion initiated by the first static pressure distribution. This is so because the outward directed flow in the boundary layer near the side walls followed the duct surface around the comer and was redirected inwards along the side walls before the static pressure distribution had reversed its orientation. This can be seen in the surface oil film visualization results, Fig. 4. Along the side wall the flow approached the $x y$-plane from top and bottom. Continuity forces the converging flow outward away from the side walls and the vortex pattern was established. The reversal in the orientation of the static pressure distribution only drove the vortices further away from the side walls. This can be seen by comparing the plots of transverse velocity components at planes 3 and 4 .

Davis and Gessner ${ }^{8}$ observed nearly identical behavior of the transverse velocitics in their measurement planes downstream of the transition region in an identical transition duct. Patrick and McCormick ${ }^{4.5}$ observed similar side wall vortices at the exit plane of their aspect ratio six duct. The side wall vortices were not apparent at the exit plane of their aspect ratio three duct, although outward directed flow in the boundary layer near the side walls, which gave rise to axial vorticity, was observed at the exit plane. Miau et al ${ }^{6,7}$ observed axial vorticity near the exit plane of their aspect ratio two transition duct which was opposite in sign to the dominant side wall vortices seen in Fig. 7.

Fig. 11 show contours of axial vorticity in the upper half of the transition duct cross section for flow without inlet swirl. The axial vorticity was calculated with a finite difference approximation using the transverse velocity components which are shown in the lower half of the cross section. Positive vorticity is represented by solid lines, negative vorticity in represented by broken lines. 
At plane 1 of Fig. 11 the initiation of the side wall vortices can be seen in the generation of positive axial vorticity in the upper right quadrant and negative axial vorticity in the upper left quadrant. This axial vorticity resulted from the static pressure gradient driven, skewinduced secondary flow in the boundary layer, as explained earlier. The sign of the axial vorticity shown in Fig. 11 is correctly predicted by Eq. (9). At plane 2 of Fig. 11 convection had carried the axial vorticity outwards towards the side walls. At plane 3 of Fig. 11 convection had carried the axial vorticity observed at plane 1 to the side walls and out of the measurement region. The new static pressure distribution, however, had generated axial vorticity which is opposite in sign from the axial vorticity observed at plane 1 . This provides additional evidence that the second static pressure distribution doesn't simply eliminate the axial vorticity created by the first static pressure distribution because convection had carried the vorticity observed at plane 1 away from the region where the opposite signed axial vorticity is produced. At plane 4 of Fig. 11 the axial vorticity observed at plane 1 is now readily apparent near the side walls, while the much weaker opposite signed axial vorticity has remained in nearly the same location as observed at plane 3.

Although there is no aerodynamic data very near the duct side wall, the surface oil film visualization provides evidence of two additional pairs of vortices in this region. This can be seen in the surface oil film visualization results presented in Fig. 4b. In the constant cross section region downstream of the transition region, a bright line appears on the duct side wall approximately 0.15 radius from the $x y$-plane. Nearby lines exhibil asymptotic behavior, approaching the bright line from either side. Fig. 12 is a sketch representing the pattern of secondary flow inferred from the surface oil film visualization. Numerical calculations at NASA Lewis Research Center using Navier-Stokes codes support this interpretation of the surface oil film visualization.

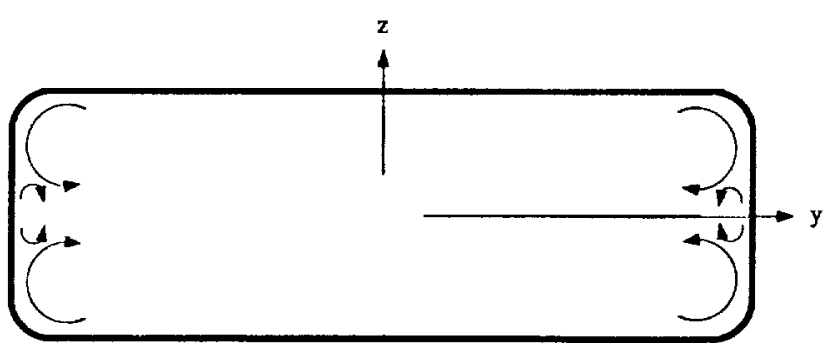

Fig. 12 The pattern of secondary

flow inferred from surface oil film visualization for flow without inlet swirl.

An effect of the vortices observed downstream of the transition region was the convection of fluid from the boundary layer into the free stream region. This produced distortion in the axial velocity and total pressure contours.
This is particularly apparent at plane 4 of Fig. 8, where the high total pressure loss fluid, normally associated with the boundary layer, extends outwards from the side wall towards the duct centerline.

There are no results which indicate any region of scparated flow in the transition duct. Davis and Gessner ${ }^{8}$ made this same observation in their study of an identical transition duct. Miau et al. ${ }^{6.7}$ observed a region of separated flow along the diverging side wall in all three of the ducts they tested, at the lowest Reynolds number condition. No separation was observed at higher Reynolds numbers. Boundary layer measurements along the diverging side walls indicated the boundary layers were laminar at their lowest Reynolds number condition and turbulent at higher Reynolds numbers, explaining the existence of the separated flow region at their low Reynolds number.

\section{Flow With Inlet Swirl}

The effect of inlet swirl on the transition duct flow field is complex. On one hand, there appears to be little influence of inlet swirl on the duct surface static pressure distribution, shown in Fig. 6. However, the difference in detailed data, in particular the transverse components of velocity in all measurement planes for flow without and with inlet swirl is striking. For example, there is no evidence in plane 3 or plane 4 of Fig. 9 of the two pair of counter-rotating side wall vortices that are observed for flow without inlet swirl.

When viewed looking downstream, the incoming flow with swirl was rotating counter clockwise, resulting in a region outside the wall boundary layer of nearly constant negative axial vorticity. The overwhelming fluid flow effect was that the duct geometry driven convergence in the $x z$-plane and the divergence in the $x y$-plane was aided by the counter clockwise swirl in the upper left and lower right quadrants and opposed by the counter clockwise swirl in the upper right and lower left quadrants. This flow pattern established the static pressure distribution for plane 1 scen in Fig. 10. The static pressure distribution again was saddle shaped, but the regions of highest static pressure were now located near the lower left and upper right comers and the regions of lowest static pressure were located near the lower right and upper left comers. The static pressure gradient associated with the maximum and minimum static pressures drove the boundary layer flow near the lower left and upper right comers in two directions, some against the direction of rotation and some towards the direction of rotation. Near the lower right and upper left comers there was no radial static pressure gradient to balance the centrifugal forces and there the flow field was directed outward. Both of these effects can be seen in the plot of transverse velocity at plane 1 of Fig. 9.

The response to the static pressure gradient was again greater in the boundary layer where the momentum was less, resulting in skew-induced secondary flows. 


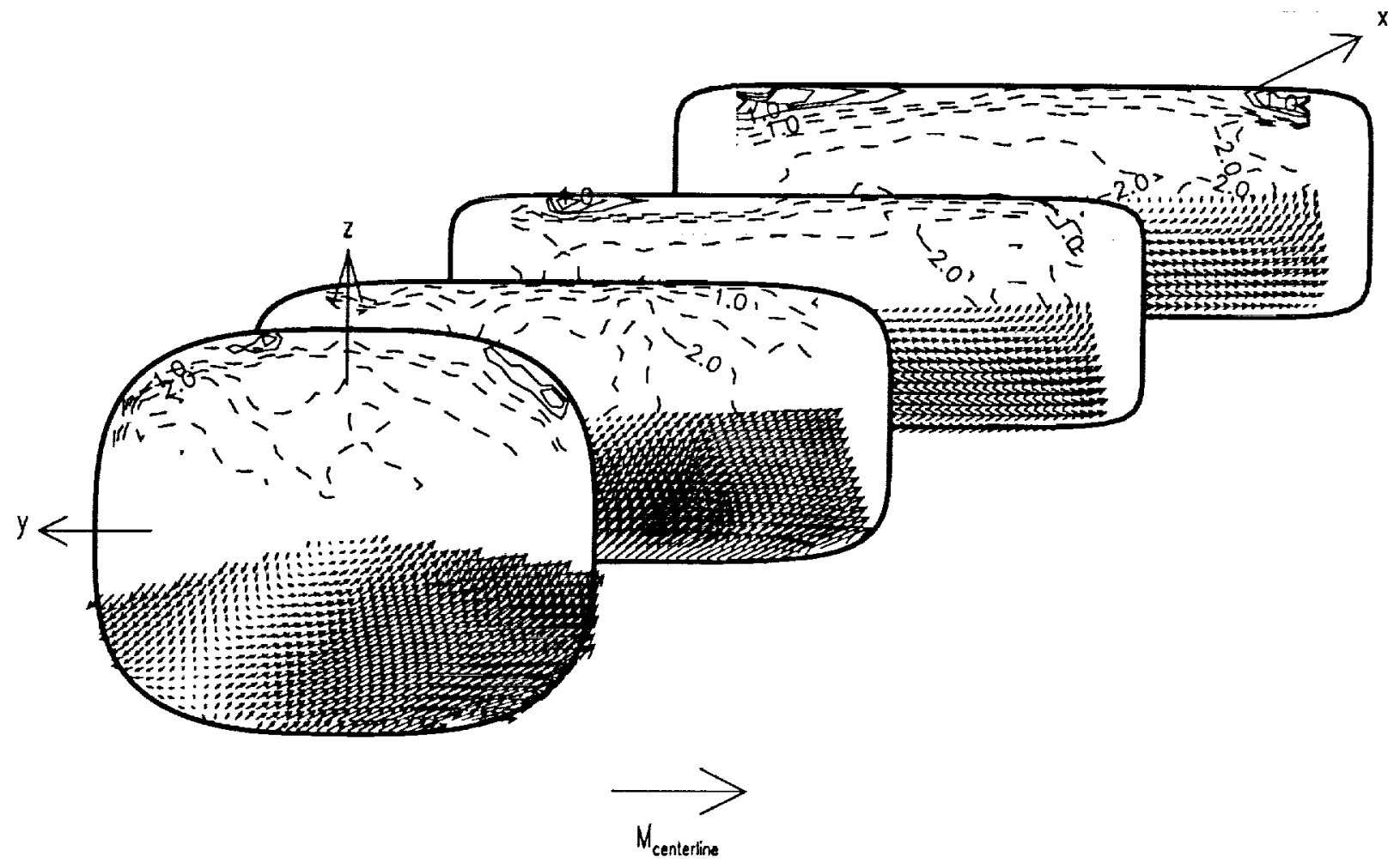

Fig. 13 Axial vorticity and transverse velocity for flow with inlet swirl.

Near the four corners this produced positive vorticity. This can be seen at plane 1 of Fig. 13, which shows axial vorticity and transverse velocity components for flow with inlet swirl. The vorticity in the upper right quadrant resulted from the static pressure gradient in the cross stream plane tangent to the surface, the vorticity in the upper left quadrant resulted from the lack of radial static pressure gradient.

The saddle-shaped static pressure distribution observed in Fig. 10 at plane 1 persisted to plane 2, however, it was not as pronounced. The complete $90^{\circ}$ change in the orientation of the static pressure distribution scen in the flow field without inlet swirl was not observed for swirling flow. Rather, the regions of highest static pressure remained in the lower left and upper right quadrants, but they appeared to be displaced clockwisc, the highest static pressures being nearer the $y$-axes and the lowest static pressures nearer the $z$-axes.

At plane 2 of Fig. 13 the positive axial vorticity observed in the upper right quadrant of plane 1 convected clockwise, against the principal direction of rotation, outwards towards the diverging side wall and out of the measurement region. This can be discerned from the surface oil film visualization shown in Fig. 5. The positive axial vorticity seen in the upper left quadrant of plane 1 also convected outwards towards the diverging side wall, but this motion is counter clockwise, with the principal direction of rotation.

At plane 3 the static pressure distribution in Fig. 10 does not differ significantly from the nonswirling static pressure distribution in the same plane. The location of regions of maximum static pressure were on the $y$-axes and the regions of minimum static pressure were located on the $z$-axes. The static pressure distribution changed its orientation nearly $90^{\circ}$ from plane 1 . The reversal of the static pressure distribution orientation for flow without inlet swirl was described earlier. For the nonswirling case, the reversal was accomplished by passing through a nearly flat static pressure distribution. For the swirling case, the reversal of the static pressure distribution orientation was accomplished without passing through a nominally flat static pressure distribution, the orientation of the static pressure distribution appeared to experience in effect a clockwise rotation.

The positive axial vorticity in the upper left quadrant of plane 3 of Fig. 13 was aided by the gradient in the static pressure distribution. The positive axial vorticity in the upper right quadrant was still out of the measurement 
region. This axial vorticity was not affected by the gradient in the static pressure distribution because of its location.

The static pressure distribution shown in plane 4 of Fig. 10 had the same shape as the distribution in plane 3, however, the gradients were not as great. At this location and further downstream, the static pressure distribution had little effect on the boundary layer flow.

Positive axial vorticity is present in each comer at plane 4 of Fig. 13. This was the same positive axial vorticity generated in each respective quadrant in plane 1. The positive vorticity in the upper left quadrant was observed in all four measurement planes. The positive vorticity in the upper right quadrant was seen at plane 1 , but had convected out of the measurement region at planes 2 and 3, and reappeared at plane 4 .

The distortion of total pressure is most apparent in the upper right and lower left quadrant in planes 3 and 4 of Fig. 10. As in the nonswirling case, this distortion resulted from the convection of boundary layer fluid by secondary flows. The slight depression in total pressure near the centerline that appeared in all four planes is an artifact of the swirl generator. All measurements indicate that the flow remained attached throughout the transition duct.

\section{Summary}

When utilized as exhaust system components of aircraft with rectangular nozzles, the incoming flow to circular-to-rectangular transition ducts often includes a swirling velocity component remaining from the gas turbine engine. Inlet swirl significantly changes the transition duct flow field. Outside the boundary layer, the response of the flow field velocity to the changing duct geometry gives rise to the static pressure distribution. For nonswirling incoming flow the static pressure distribution produced skew-induced secondary flows within the boundary layer which evolved into two pair of counterrotating side wall vortices. For the inlet swirl case studied the side wall vortices were not observed. The static pressure distribution was altered by the swirling velocity flow ficld to an extent that the side wall vortices were suppressed. The effects of inlet swirl should be included in the design of circular-to-rectangular transition ducts for aircraft exhaust systems.

\section{References}

${ }^{1}$ Whitney, W. J., Schum, H. J., and Behning, F. P., "ColdAir Investigation of a Turbine for High-TemperatureEngine Application IV - Two-Stage Turbine Performance," NASA TN D-6960, 1972.
${ }^{2}$ S7anca, E. M., Schum, H. J., and Holz, G. M., "Research Turbine for High-Temperature Core Engine Application I - Cold-Air Overall Performance of Solid Scalcd Turbine," NASA TN D-7557, 1974.

${ }^{3}$ Schwab, J. R., Stabe, R. G., and Whitney, W. J., "Analytical and Experimental Study of Flow Through Axial Turbine Stage With a Nonuniform Inlet Radial Temperature Profile," AIAA Paper 83-1175, 1983.

${ }^{4}$ Patrick, W. P. and McCormick, D. C., "Laser Velocimeter and Total Pressure Measurements in Circularto-Rectangular Transition Ducts," United Technologies Research Center Report 87-41, June 1988. NASA CR to be published.

${ }^{5}$ Patrick, W. P. and McCormick, D. C., "Circular-toRectangular Duct Flows - A Benchmark Experimental Study," Socicly of Automotive Engineers Tech. Rep. 871776, 1988.

${ }^{6}$ Miau, J. J., Lin, S. A., Chou, J. H., Wei, C. Y., and Lin, C. K., "An Experimental Study of Flow in a Circular-Rectangular Transition Duct," AIAA Paper 883029, 1988.

${ }^{7}$ Miau, J. J., Leu, T. S., Chou, J. H., Lin, S. A., and Lin, C. K., "Flow Distortion in a Circular-to-Rectangular Transition Duct," AIAA Journal, Vol. 28, Aug. 1990, pp. 1447-1456.

${ }^{8}$ Davis, D. O. and Gessner, F. B., "Experimental Investigation of Turbulent Flow Through a Circular-toRectangular Transition Duct," AIAA Paper 90-1505, 1990.

${ }^{9}$ J. R. Burley, J. and Carlson, J. R., "Circular-toRectangular Transition Ducts for High-Aspect Ratio Nonaxisymmetric Nozzles," AIAA Paper 85-1346, 1985.

${ }^{10}$ J. R. Burley, J., Bangert, L. S., and Carlson, J. R., "Investigation of Circular-to-Rectangular Transition Ducts for High-Aspect Ratio Nonaxisymmetric Nozzles," NASA TP 2534, Mar. 1986.

${ }^{11}$ Reichert, B. A., A Study of High Speed Flows in an Aircraft Transition Duct. PhD thesis, Iowa State University, Ames, Iowa, 1991.

${ }^{12}$ Squire, L. C., Maltby, R. L., Keating, R. F. A., and Stanbrook, A., "The Surface Oil Flow Technique," in Flow Visualization in Wind Tunnels Using Indicators (Maltby, R. L., ed.), pp. 1-28, AGARD, Apr. 1962. AGARDograph 70.

${ }^{13}$ Jurkovich, M. S., Greber, I., and Hingst, W. R., "Flow Visualization Studies of a 3-D Shock/Boundary Layer Interaction in the Presence of a Non-Uniform Approach Boundary Layer," AIAA Paper 84-1560, 1984.

${ }^{14}$ Bradshaw, P., "Turbulent Secondary Flows," Annual Review of Fluid Mechanics, Vol. 19, 1987, pp. 53-74. 


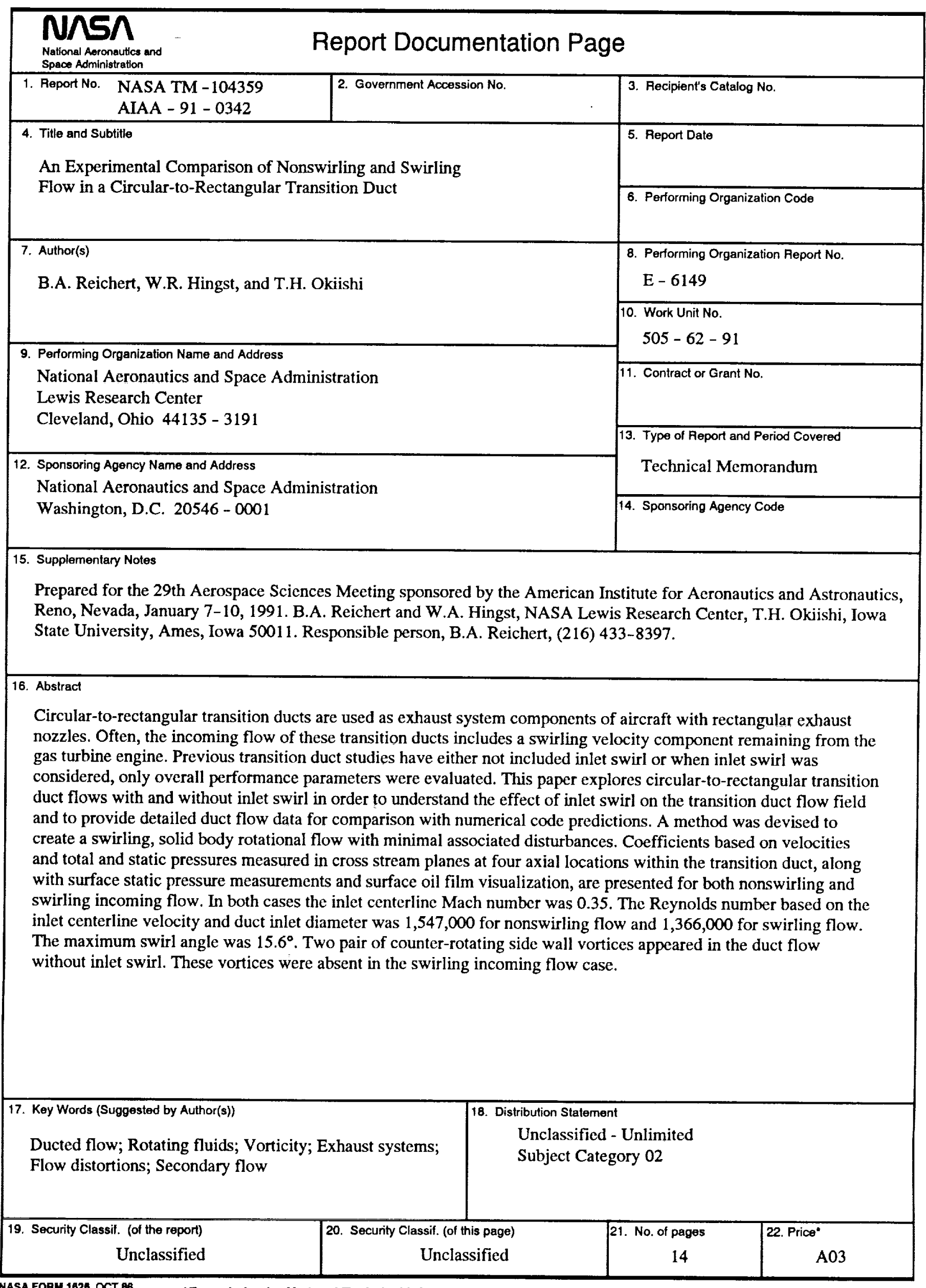

